\title{
Particle Swarm Optimization for Design of Beam
}

\author{
[Pooja Ashok Yerekar ${ }^{1}$, Rahul D. Pandit ${ }^{2}$, Dr. A. P. Wadekar ${ }^{3}$ ]
}

\begin{abstract}
Particle Swarm Optimization is an optimization technique emulates from social behaviour i.e. birds flocking, fish schooling and bee's behaviour and it is also related to A-life i.e. artificial life (human life). It has wide range of applications in almost every field. In this paper we are applying this technique to Civil Engineering field for designing different types of beam loaded with UDL and supported with different support conditions i.e. simply support, cantilever and fixed support for obtaining optimal quantities of area of steel, moment of resistance and shear stress. With the help of PSO algorithm, MAT-LAB coding is formulated. Area of steel, Moment of resistance and Shear Stress obtain with the help of PSO model is compared with the results obtain by different computational methods i.e. 1) By using IS 456-2000 with manual calculations by rounding off values; 2) Using IS 456-2000 with manual calculations without rounding off values, And 3) Excel sheet calculation. (Abstract)
\end{abstract}

Keywords-GBEST - Global Best, LBEST - Local Best, PSO - Particle Swarm Optimization, R/F reinforcement (key words)

\section{Introduction}

Optimization is the process of obtaining the best result under given circumstances to minimize the efforts required or to maximize the desired benefits of analysis. While designing any structure designer needs to assume some values about properties of materials under safety criteria. There are also a one more criteria, economical criteria. Particle Swarm optimization method helps us to maintain the balance situation in between those criteria's. It is related to evolutionary computation, and has ties to both genetic algorithms (GA) and evolutionary programming.

Particle Swarm optimization (PSO) method is proposed by Kennedy and Eberhart (1995). It is obtain from social model that don't have leader in

\footnotetext{
Pooja Ashok Yerekar $^{1}$ (PG Student)

PES College (BAMU)

INDIA

Rahul D. Pandit ${ }^{2}$ (Assistant Professor)

Dr. A. P. Wadekar ${ }^{3}$ (Principal in Grade Professor)
}

their group by studying birds flocking, bee's behaviour and fish schooling in particular. Typically, a flock of animal that have no leader will find food by random, follow one of the members of the group that has the closest position with a food source (potential solution). The flock achieves their best condition simultaneously through communication among members who already have a better situation. Animal which has a better condition will inform it to its flocks and the others will move simultaneously to that place. This would happen repeatedly until the best conditions or a food source has discovered.

\section{Literature Survey}

PSO approach is in use for optimization of nonlinear function, developed by social metaphor. It has ties to A-life, bird flocking, fish schooling, and swarming theory in particular and also related to GA \& evolutionary programming. PSO is effectively simple algorithm use for many engineering and scientific research optimization field [1]. In practice mostly we use under-reinforced section design. PSO gives us a design of beam as an under-reinforced section which is safer from design criteria given by IS-456 2000. It also provides economical sectional design of beam with provision of less area of steel as compared to area of steel required by manual calculation by IS456-2000. PSO reduces the efforts required to find the optimal and safe design results with great number of trials [2]. To improve the convergence speed with best quality solution speed of PSO follows the biologically inspired computational search optimization method based on stochastic evolutionary optimization method. To deal with the problems of PSO, basic PSO method is present. Modified variant PSO is use to overcome the defects of basic PSO. Numbers of variants are in use to improve the performance of this method [3,4]. PSO algorithm is use for structural field for optimization with tuning parameters to promote best global/local search for cut off premature convergence. This is an idea method in structural engineering field due to its simplicity in implementation and better ability for optimization with compare to other optimization field [5]. Accordingly 'No Free Lunch' theorem only single method is not enough for solving optimization 
Proc. of the Eighth Intl. Conf. on Advances in Civil, Structural and Environmental Engineering - ACSEE 2019

Copyright (C) Institute of Research Engineers and Doctors. All rights reserved.

ISBN: 978-1-63248-166-5 DOI : 10.15224/978-1-63248-166-5-01

problem effectively. EPSO is use as an effective and powerful method for solving different types of complex optimization problem without getting trap into local optima [6]. Due to subtraction based solution mechanism diversity in particle population is lost, showing the stagnation behavior and to deal with this problem 'Velocity and Position Update' rule is useful [7]. Numbers of iterations get affected due to changes in paradigms. GBEST performs best in medium numbers of iterations while LBEST with two numbers of iterations is most exact [8]. PSO's performance is not sensitive to population size and has ability to converge toward optimal result. Due to linearly decreasing inertia weight it has lack the global search ability at the run's end, which fails to find optimal value of complicated problems. By employing self adapting approach this difficulty can be overcome [9]. Studies of human cognitive psychology shows, best planer regulate their strategies with respect to the current state and their perception of the experience from others. From this learning strategies SRPSO is invented, which performs better than other PSO variants with 95\% confidence level [10]. Design of beam is a heuristic optimization problem can be solve effectively by proper allocation of constraint based on engineering clauses given by different IS codes with exact objective function. With the help of PSO large size design problems can be solve with this technique with less efforts and effective optimization results [11]. To solve large size complex problems, TSP is use with uncertainty and crossover technique t speed up convergence speed [12]. PSO technique can replace GA and other optimization technique for beam-slab design with perfect solution [13].

\section{Methodology}

By simulating the social model 'B. K. Chakrabarty' has developed a model for Optimal Design of Reinforced Concrete beam for cost optimization. With reference to that model I have created 3 models for design of Reinforced concrete Beam with different support conditions i.e. Simply Support, Cantilever and Fixed respectively loaded with UDL. Algorithm 1 describes the details of algorithm use for simply supported beam formed by using IS Code clauses. Algorithm 2 describes the details of algorithm use for cantilever beam formed by using IS Code clauses for safe design. Algorithm 3 describes the details of algorithm use for fixed beam formed with IS Code design clauses. 
Proc. of the Eighth Intl. Conf. on Advances in Civil, Structural and Environmental Engineering - ACSEE 2019

Copyright (C) Institute of Research Engineers and Doctors. All rights reserved.

ISBN: 978-1-63248-166-5 DOI : 10.15224/978-1-63248-166-5-01

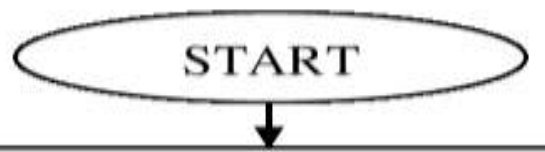

Constraint 1: Deflection Constraint

$\mathrm{a}_{1} \mathrm{a}_{2}-\mathrm{x}_{1} \leq \mathrm{O}$

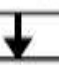

Constraint 2: Total Depth Constraint

$$
\mathrm{x}_{1}-\mathrm{x}_{2}+\mathrm{a}_{3} \leq \mathrm{O}
$$

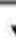

Constraint 3: Loading Constraint

$$
\mathrm{x}_{4}-\mathrm{x}_{5}+\mathrm{a}_{6}+\mathrm{a}_{7}=0
$$

Constraint 4: Moment constraint

$$
\mathrm{x}_{6}=\left[1.5\left(\mathrm{WL}^{2}\right)\right] / 8
$$

Constraint 5: Shear Force constraint

$$
\mathrm{x}_{7}=[1.5(\mathrm{WL})] / 2
$$

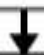

Constraint 6: Under R/F constraint

$$
\mathrm{X}_{8}=\mathrm{a}_{8} \text { ag } \mathrm{x}_{3} \mathrm{x}_{1}{ }^{2}
$$

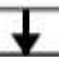

Constraint 7: Area of Steel constraint

$$
\mathrm{x}_{9}=\mathrm{a}_{9} \mathrm{a}_{10} \mathrm{x}_{1} \mathrm{x}_{3} \mathrm{a}_{11}{ }^{-1}\left(1-\mathrm{a}_{13}\right)
$$

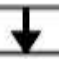

\section{Constraint 8: Minimum R/F constraint}

$$
\mathrm{x}_{10}=\mathrm{a}_{14} \mathrm{x}_{3} \mathrm{x}_{1} \mathrm{a}_{11}{ }^{-1}
$$

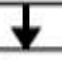

Constraint 9: Maximum $\mathrm{R} / \mathrm{F}$ constraint

$$
\mathrm{x}_{11}=\mathrm{a}_{15} \mathrm{x}_{2} \mathrm{x}_{3}
$$

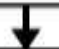

Constraint 10: Shear Stress constraint

$$
x_{12}=x_{7} x_{1}^{-1} x_{3}^{-1}
$$

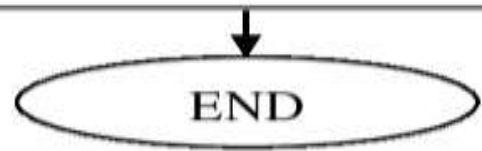

Algorithm 1. Algorithm for Simply Supported Beam 
Proc. of the Eighth Intl. Conf. on Advances in Civil, Structural and Environmental Engineering - ACSEE 2019

Copyright (C) Institute of Research Engineers and Doctors. All rights reserved.

ISBN: 978-1-63248-166-5 DOI : 10.15224/978-1-63248-166-5-01

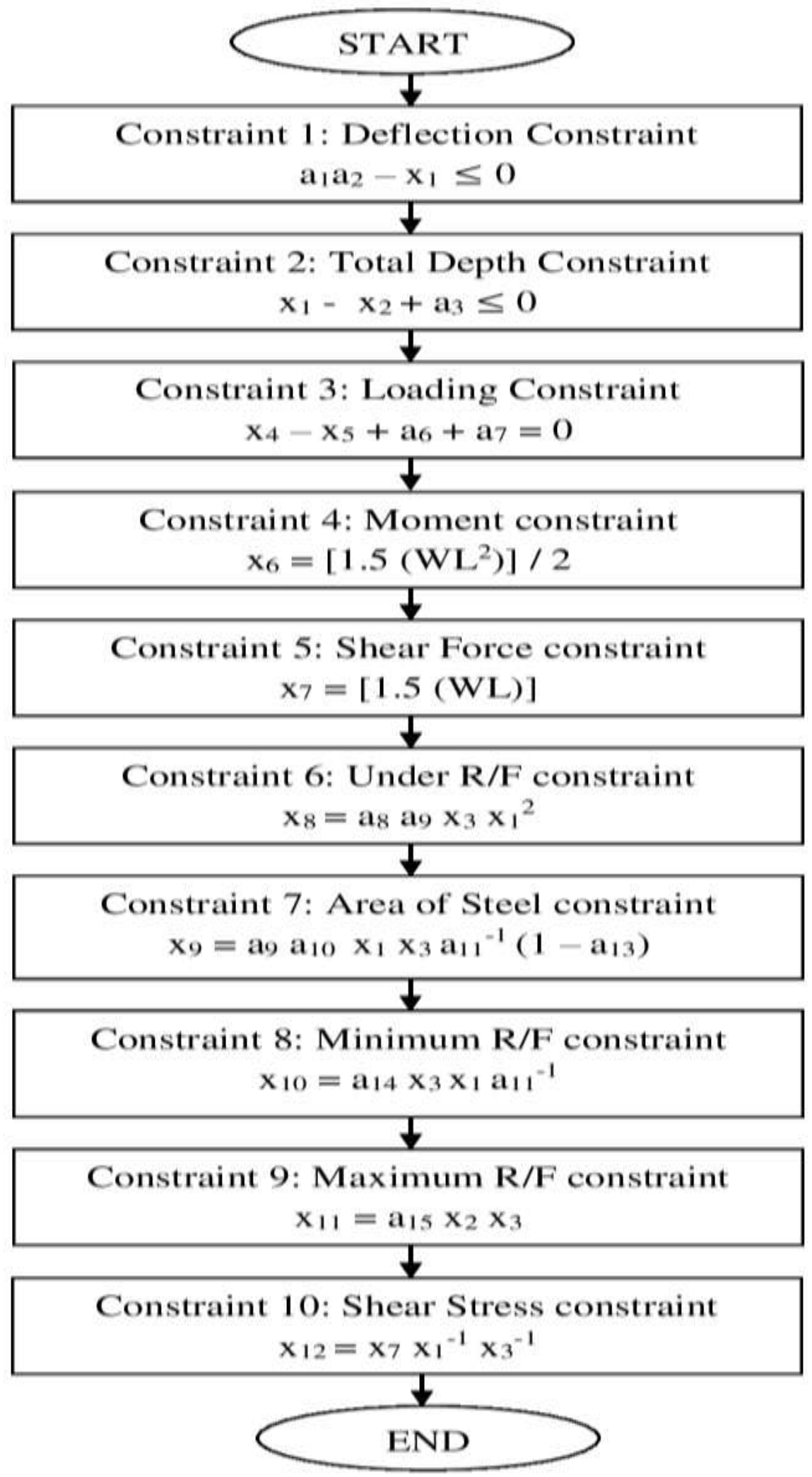

Algorithm 2. Algorithm for Cantilever Beam 
Proc. of the Eighth Intl. Conf. on Advances in Civil, Structural and Environmental Engineering - ACSEE 2019

Copyright (C) Institute of Research Engineers and Doctors. All rights reserved.

ISBN: 978-1-63248-166-5 DOI : 10.15224/978-1-63248-166-5-01

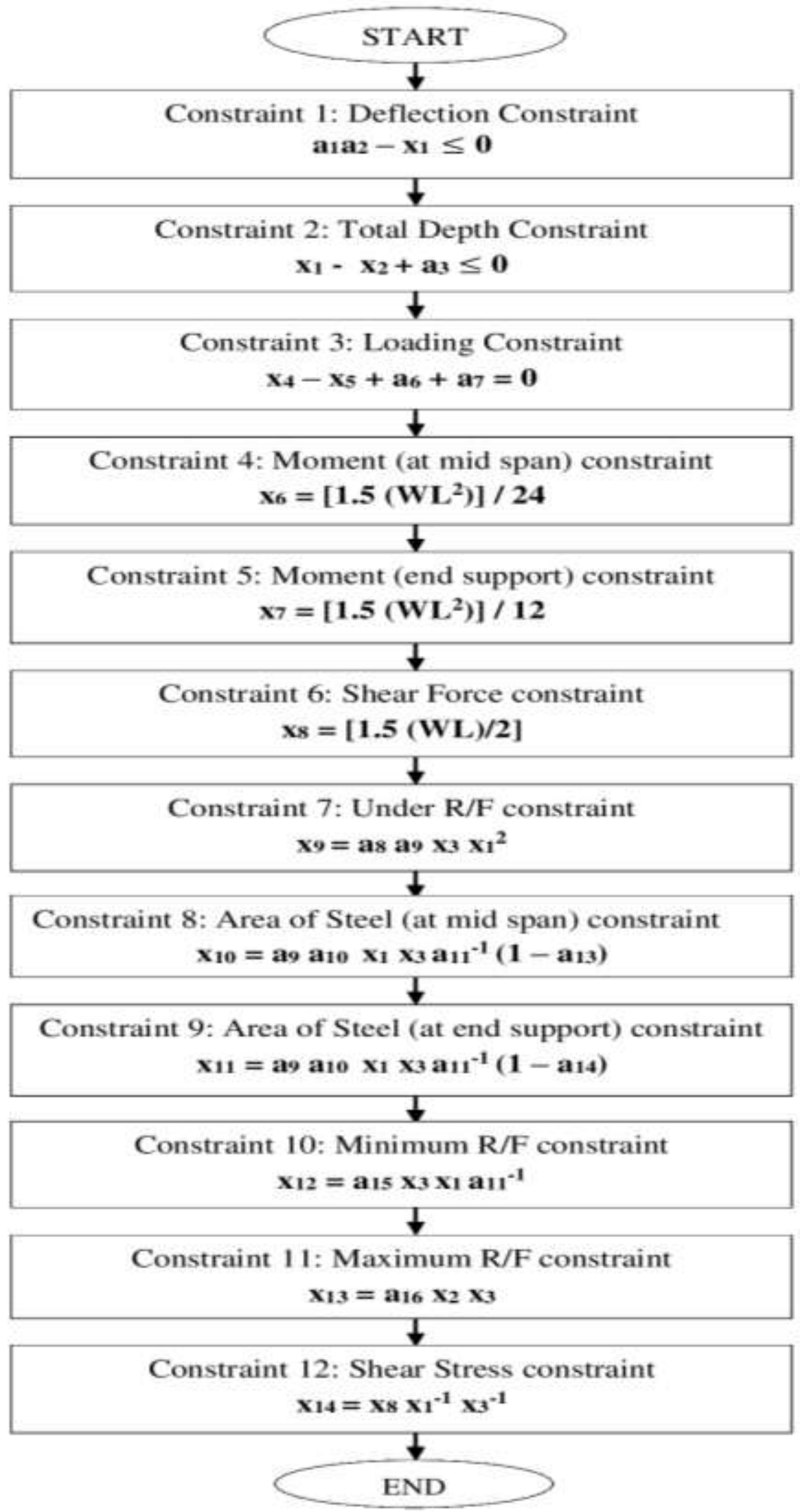

Algorithm 3. Algorithm for Fixed Beam 
Proc. of the Eighth Intl. Conf. on Advances in Civil, Structural and Environmental Engineering - ACSEE 2019

Copyright $\odot$ Institute of Research Engineers and Doctors. All rights reserved.

ISBN: 978-1-63248-166-5 DOI : 10.15224/978-1-63248-166-5-01

With the help of above algorithms by collaborating with PSO algorithm, MATLAB algorithm created to find the result by PSO approach.

\section{Results}

Results obtained by PSO method is compared with method 1 (manual calculations done using IS456 2000 by rounding off values), method 2 (manual calculations done by using IS456-2000 without rounding off values) and method 3 (Excel sheet calculations). Results obtained are tabulated as follows with units: Area of steel in $\mathrm{mm}^{2}$, Moment of Resistance in $\mathrm{KN} \cdot \mathrm{m}$ and Shear Stress in $\mathrm{KN} / \mathrm{mm}^{2}$.

TABLE I. Simply Supported Beam's Result

\begin{tabular}{|c|c|c|c|c|c|}
\hline Sr. No. & Particular & Method 1 & Method 2 & Method 3 & PSO method \\
\hline \multirow{3}{*}{1.} & Area of steel & 338.43 & 410.84 & 401.90 & 330.00 \\
\hline & Moment of Resistance & 32.910 & 31.570 & 31.000 & 33.070 \\
\hline & Shear Stress & 0.4000 & 0.4000 & 0.0000 & 0.3800 \\
\hline \multirow{3}{*}{2.} & Area of steel & 331.33 & 408.06 & 398.40 & 320.00 \\
\hline & Moment of Resistance & 31.760 & 30.580 & 30.000 & 31.960 \\
\hline & Shear Stress & 0.4000 & 0.4000 & 0.0000 & 0.3900 \\
\hline \multirow{3}{*}{3.} & Area of steel & 327.33 & 408.64 & 392.47 & 315.00 \\
\hline & Moment of Resistance & 30.980 & 29.920 & 29.000 & 31.030 \\
\hline & Shear Stress & 0.4000 & 0.5000 & 0.0000 & 0.3900 \\
\hline \multirow{3}{*}{4.} & Area of steel & 482.96 & 518.75 & 513.40 & 477.00 \\
\hline & Moment of Resistance & 48.178 & 47.400 & 47.000 & 49.020 \\
\hline & Shear Stress & 0.4000 & 0.4000 & 0.0000 & 0.3900 \\
\hline \multirow{3}{*}{5.} & Area of steel & 475.07 & 513.64 & 503.25 & 470.05 \\
\hline & Moment of Resistance & 46.410 & 45.730 & 45.000 & 47.010 \\
\hline & Shear Stress & 0.5000 & 0.5000 & 0.0000 & 0.4900 \\
\hline \multirow{3}{*}{6.} & Area of steel & 471.83 & 513.42 & 491.34 & 462.00 \\
\hline & Moment of Resistance & 45.225 & 44.617 & 44.000 & 45.690 \\
\hline & Shear Stress & 0.5000 & 0.5000 & 0.0000 & 0.4000 \\
\hline \multirow{3}{*}{7.} & Area of steel & 598.17 & 635.99 & 634.11 & 582.00 \\
\hline & Moment of Resistance & 68.220 & 67.160 & 67.000 & 68.850 \\
\hline & Shear Stress & 0.4000 & 0.4000 & 0.0000 & 0.4000 \\
\hline \multirow{3}{*}{8.} & Area of steel & 586.82 & 627.83 & 621.04 & 575.00 \\
\hline & Moment of Resistance & 65.460 & 64.540 & 64.000 & 66.010 \\
\hline & Shear Stress & 0.5000 & 0.5000 & 0.0000 & 0.4000 \\
\hline \multirow{3}{*}{9.} & Area of steel & 602.59 & 639.70 & 639.36 & 592.00 \\
\hline & Moment of Resistance & 69.130 & 68.030 & 68.000 & 69.850 \\
\hline & Shear Stress & 0.5000 & 0.5000 & 0.0000 & 0.4000 \\
\hline \multirow{3}{*}{10.} & Area of steel & 722.78 & 762.85 & 761.06 & 715.00 \\
\hline & Moment of Resistance & 92.540 & 91.170 & 91.000 & 92.870 \\
\hline & Shear Stress & 0.5000 & 0.5000 & 0.0000 & 0.4900 \\
\hline \multirow{3}{*}{11.} & Area of steel & 735.56 & 773.68 & 766.04 & 728.00 \\
\hline & Moment of Resistance & 95.280 & 93.750 & 93.000 & 95.650 \\
\hline & Shear Stress & 0.5000 & 0.5000 & 0.0000 & 0.4000 \\
\hline \multirow{3}{*}{12.} & Area of steel & 707.59 & 751.12 & 747.72 & 699.00 \\
\hline & Moment of Resistance & 88.500 & 87.300 & 87.000 & 89.500 \\
\hline & Shear Stress & 0.5000 & 0.5000 & 0.0000 & 0.4000 \\
\hline \multirow{3}{*}{13.} & Area of steel & 687.61 & 773.67 & 766.04 & 690.00 \\
\hline & Moment of Resistance & 97.500 & 93.750 & 93.000 & 98.020 \\
\hline & Shear Stress & 0.4000 & 0.4000 & 0.0000 & 0.4000 \\
\hline \multirow{3}{*}{14.} & Area of steel & 673.64 & 762.85 & 761.06 & 666.00 \\
\hline & Moment of Resistance & 94.620 & 91.17 & 91.000 & 94.660 \\
\hline & Shear Stress & 0.4000 & 0.4000 & 0.0000 & 0.4000 \\
\hline \multirow{3}{*}{15.} & Area of steel & 652.32 & 751.12 & 747.72 & 650.00 \\
\hline & Moment of Resistance & 90.000 & 87.300 & 87.000 & 90.060 \\
\hline & Shear Stress & 0.5000 & 0.5000 & 0.0000 & 0.4600 \\
\hline \multirow{3}{*}{16.} & Area of steel & 873.42 & 913.87 & 910.25 & 860.00 \\
\hline & Moment of Resistance & 125.29 & 123.39 & 123.00 & 126.03 \\
\hline & Shear Stress & 0.5000 & 0.5000 & 0.0000 & 0.5000 \\
\hline \multirow{3}{*}{17.} & Area of steel & 900.03 & 938.59 & 930.94 & 900.23 \\
\hline & Moment of Resistance & 130.99 & 128.86 & 128.00 & 131.25 \\
\hline & Shear Stress & 0.4000 & 0.4000 & 0.0000 & 0.4000 \\
\hline
\end{tabular}


Proc. of the Eighth Intl. Conf. on Advances in Civil, Structural and Environmental Engineering - ACSEE 2019

Copyright $\odot$ Institute of Research Engineers and Doctors. All rights reserved. ISBN: 978-1-63248-166-5 DOI : 10.15224/978-1-63248-166-5-01

\begin{tabular}{|c|c|c|c|c|c|}
\hline \multirow{3}{*}{18.} & Area of steel & 889.87 & 913.87 & 910.25 & 872.00 \\
\hline & Moment of Resistance & 124.38 & 123.39 & 123.00 & 124.39 \\
\hline & Shear Stress & 0.5000 & 0.5000 & 0.0000 & 0.5000 \\
\hline \multirow{3}{*}{19.} & Area of steel & 878.09 & 899.69 & 892.49 & 875.09 \\
\hline & Moment of Resistance & 120.66 & 119.75 & 119.00 & 121.00 \\
\hline & Shear Stress & 0.5000 & 0.5000 & 0.0000 & 0.5000 \\
\hline \multirow{3}{*}{20.} & Area of steel & 857.49 & 899.69 & 892.49 & 859.49 \\
\hline & Moment of Resistance & 121.50 & 119.75 & 119.00 & 121.30 \\
\hline & Shear Stress & 0.5000 & 0.5000 & 0.0000 & 0.4000 \\
\hline \multirow{3}{*}{21.} & Area of steel & 890.88 & 930.02 & 929.66 & 888.00 \\
\hline & Moment of Resistance & 129.09 & 127.04 & 127.00 & 129.97 \\
\hline & Shear Stress & 0.5000 & 0.5000 & 0.0000 & 0.5000 \\
\hline
\end{tabular}

TABLE II. Cantilever Beam's Result

\begin{tabular}{|c|c|c|c|c|c|}
\hline Sr. No. & Particular & Method 1 & Method 2 & Method 3 & PSO method \\
\hline \multirow{3}{*}{1.} & Area of steel & 226.60 & 235.75 & 175.00 & 220.00 \\
\hline & Moment of Resistance & 023.25 & 22.920 & 15.000 & 23.650 \\
\hline & Shear Stress & 0.3000 & 0.2000 & 0.0000 & 0.3000 \\
\hline \multirow{3}{*}{2.} & Area of steel & 216.80 & 235.59 & 175.00 & 212.98 \\
\hline & Moment of Resistance & 23.025 & 22.905 & 15.000 & 23.950 \\
\hline & Shear Stress & 0.2000 & 0.3000 & 0.0000 & 0.2000 \\
\hline \multirow{3}{*}{3.} & Area of steel & 329.74 & 238.45 & 186.00 & 319.58 \\
\hline & Moment of Resistance & 24.255 & 23.433 & 15.000 & 25.000 \\
\hline & Shear Stress & 0.2000 & 0.3000 & 0.0000 & 0.2000 \\
\hline \multirow{3}{*}{4.} & Area of steel & 392.62 & 406.84 & 262.00 & 385.98 \\
\hline & Moment of Resistance & 59.910 & 58.790 & 34.000 & 59.990 \\
\hline & Shear Stress & 0.3000 & 0.3000 & 0.0000 & 0.3000 \\
\hline \multirow{3}{*}{5.} & Area of steel & 402.65 & 416.90 & 280.00 & 398.00 \\
\hline & Moment of Resistance & 61.590 & 60.410 & 34.000 & 61.520 \\
\hline & Shear Stress & 0.3000 & 0.3000 & 0.0000 & 0.3000 \\
\hline \multirow{3}{*}{6.} & Area of steel & 389.64 & 431.93 & 306.00 & 369.00 \\
\hline & Moment of Resistance & 67.060 & 62.810 & 34.000 & 67.950 \\
\hline & Shear Stress & 0.3000 & 0.3000 & 0.0000 & 0.3000 \\
\hline \multirow{3}{*}{7.} & Area of steel & 66.060 & 93.400 & 81.480 & 65.230 \\
\hline & Moment of Resistance & 4.5500 & 4.3800 & 3.0000 & 5.0300 \\
\hline & Shear Stress & 0.3000 & 0.4100 & 0.0000 & 0.2000 \\
\hline \multirow{3}{*}{8.} & Area of steel & 67.570 & 94.270 & 80.230 & 60.000 \\
\hline & Moment of Resistance & 4.6870 & 4.4900 & 3.0000 & 4.9500 \\
\hline & Shear Stress & 0.2000 & 0.3000 & 0.0000 & 0.2000 \\
\hline \multirow{3}{*}{9.} & Area of steel & 56.410 & 90.980 & 79.640 & 59.520 \\
\hline & Moment of Resistance & 4.9700 & 4.3800 & 3.0000 & 5.0200 \\
\hline & Shear Stress & 0.2000 & 0.3000 & 0.0000 & 0.2000 \\
\hline \multirow{3}{*}{10.} & Area of steel & 111.43 & 151.72 & 118.84 & 106.00 \\
\hline & Moment of Resistance & 11.600 & 10.870 & 8.0000 & 11.870 \\
\hline & Shear Stress & 0.3000 & 0.3000 & 0.0000 & 0.3000 \\
\hline \multirow{3}{*}{11.} & Area of steel & 108.05 & 153.83 & 118.39 & 101.00 \\
\hline & Moment of Resistance & 12.080 & 11.090 & 8.0000 & 12.120 \\
\hline & Shear Stress & 0.2000 & 0.3000 & 0.0000 & 0.2000 \\
\hline \multirow{3}{*}{12.} & Area of steel & 105.53 & 151.72 & 118.83 & 103.00 \\
\hline & Moment of Resistance & 11.770 & 10.870 & 8.0000 & 11.980 \\
\hline & Shear Stress & 0.2000 & 0.3000 & 0.0000 & 0.2000 \\
\hline \multirow{3}{*}{13.} & Area of steel & 106.83 & 152.91 & 118.46 & 104.00 \\
\hline & Moment of Resistance & 11.930 & 10.990 & 8.0000 & 12.000 \\
\hline & Shear Stress & 0.2200 & 0.3200 & 0.0000 & 0.2000 \\
\hline \multirow{3}{*}{14.} & Area of steel & 114.50 & 155.09 & 117.84 & 112.00 \\
\hline & Moment of Resistance & 12.240 & 11.210 & 8.0000 & 12.950 \\
\hline & Shear Stress & 0.2000 & 0.3000 & 0.0000 & 0.2000 \\
\hline \multirow{3}{*}{15.} & Area of steel & 114.00 & 153.88 & 118.14 & 109.00 \\
\hline & Moment of Resistance & 11.900 & 11.098 & 8.0000 & 11.980 \\
\hline & Shear Stress & 0.2000 & 0.3000 & 0.0000 & 0.3000 \\
\hline \multirow{4}{*}{16.} & Area of steel & 290.60 & 316.85 & 219.00 & 279.00 \\
\hline & Moment of Resistance & 39.840 & 38.330 & 24.000 & 40.010 \\
\hline & Shear Stress & 0.3000 & 0.3000 & 0.0000 & 0.2900 \\
\hline & Area of steel & 299.15 & 313.75 & 219.00 & 285.00 \\
\hline
\end{tabular}


Proc. of the Eighth Intl. Conf. on Advances in Civil, Structural and Environmental Engineering - ACSEE 2019

Copyright $\odot$ Institute of Research Engineers and Doctors. All rights reserved.

ISBN: 978-1-63248-166-5 DOI : 10.15224/978-1-63248-166-5-01

\begin{tabular}{|c|c|c|c|c|c|}
\hline \multirow{2}{*}{17.} & Moment of Resistance & 38.790 & 37.980 & 24.000 & 39.000 \\
\hline & Shear Stress & 0.3000 & 0.3000 & 0.0000 & 0.3000 \\
\hline \multirow{3}{*}{18.} & Area of steel & 297.69 & 323.81 & 233.00 & 282.00 \\
\hline & Moment of Resistance & 40.898 & 39.280 & 24.000 & 41.030 \\
\hline & Shear Stress & 0.3000 & 0.3000 & 0.0000 & 0.2800 \\
\hline \multirow{3}{*}{19.} & Area of steel & 305.99 & 320.56 & 233.00 & 302.00 \\
\hline & Moment of Resistance & 39.770 & 38.910 & 24.000 & 39.990 \\
\hline & Shear Stress & 0.3000 & 0.3000 & 0.0000 & 0.3000 \\
\hline \multirow{3}{*}{20.} & Area of steel & 287.20 & 323.81 & 233.00 & 276.00 \\
\hline & Moment of Resistance & 41.600 & 39.280 & 24.000 & 41.750 \\
\hline & Shear Stress & 0.2000 & 0.3000 & 0.0000 & 0.2000 \\
\hline \multirow{3}{*}{21.} & Area of steel & 284.91 & 334.61 & 255.00 & 280.00 \\
\hline & Moment of Resistance & 44.530 & 40.730 & 24.000 & 44.980 \\
\hline & Shear Stress & 0.2000 & 0.3000 & 0.0000 & 0.2000 \\
\hline
\end{tabular}

TABLE III. Fixed Beam’s Result

\begin{tabular}{|c|c|c|c|c|c|}
\hline $\begin{array}{l}\text { Sr. } \\
\text { No }\end{array}$ & Particular & Method 1 & Method 2 & Method 3 & $\begin{array}{c}\text { PSO } \\
\text { method }\end{array}$ \\
\hline \multirow{5}{*}{1.} & Area of steel at mid span & 141.32 & 121.97 & 117.00 & 141.02 \\
\hline & Area of steel at support & 216.76 & 257.06 & 256.52 & 210.78 \\
\hline & Moment of Resistance at mid span & 10.970 & 10.520 & 10.000 & 11.030 \\
\hline & Moment of Resistance at support & 21.940 & 21.040 & 21.000 & 22.000 \\
\hline & Shear Stress & 0.4000 & 0.4000 & 0.0000 & 0.3000 \\
\hline \multirow{5}{*}{2.} & Area of steel at mid span & 122.89 & 118.80 & 116.47 & 120.66 \\
\hline & Area of steel at support & 210.93 & 252.46 & 247.00 & 201.00 \\
\hline & Moment of Resistance at mid span & 10.590 & 10.190 & 10.000 & 10.980 \\
\hline & Moment of Resistance at support & 21.170 & 20.390 & 20.000 & 21.200 \\
\hline & Shear Stress & 0.4000 & 0.4000 & 0.0000 & 0.4000 \\
\hline \multirow{5}{*}{3.} & Area of steel at mid span & 110.60 & 116.80 & 104.82 & 101.00 \\
\hline & Area of steel at support & 207.36 & 249.93 & 236.36 & 198.00 \\
\hline & Moment of Resistance at mid span & 10.330 & 9.9700 & 9.0000 & 10.980 \\
\hline & Moment of Resistance at support & 20.660 & 19.950 & 19.000 & 21.060 \\
\hline & Shear Stress & 0.4000 & 0.5000 & 0.0000 & 0.4000 \\
\hline \multirow{5}{*}{4.} & Area of steel at mid span & 150.75 & 152.981 & 141.00 & 140.00 \\
\hline & Area of steel at support & 304.24 & 323.32 & 316.46 & 298.03 \\
\hline & Moment of Resistance at mid span & 16.060 & 15.800 & 15.000 & 16.950 \\
\hline & Moment of Resistance at support & 32.120 & 31.600 & 31.000 & 32.980 \\
\hline & Shear Stress & 0.4000 & 0.4000 & 0.0000 & 0.3000 \\
\hline \multirow{5}{*}{5.} & Area of steel at mid span & 140.35 & 148.38 & 145.92 & 142.29 \\
\hline & Area of steel at support & 296.42 & 316.09 & 310.44 & 297.00 \\
\hline & Moment of Resistance at mid span & 15.470 & 15.240 & 15.000 & 15.980 \\
\hline & Moment of Resistance at support & 30.940 & 30.480 & 30.000 & 30.980 \\
\hline & Shear Stress & 0.5000 & 0.5000 & 0.0000 & 0.4000 \\
\hline \multirow{5}{*}{6.} & Area of steel at mid span & 137.34 & 145.48 & 136.47 & 140.00 \\
\hline & Area of steel at support & 291.75 & 312.14 & 303.19 & 293.52 \\
\hline & Moment of Resistance at mid span & 15.075 & 14.870 & 14.000 & 15.080 \\
\hline & Moment of Resistance at support & 30.150 & 29.740 & 29.000 & 30.250 \\
\hline & Shear Stress & 0.5000 & 0.5000 & 0.0000 & 0.5000 \\
\hline \multirow{5}{*}{7.} & Area of steel at mid span & 178.04 & 186.12 & 182.80 & 175.00 \\
\hline & Area of steel at support & 374.89 & 394.59 & 386.96 & 370.00 \\
\hline & Moment of Resistance at mid span & 22.740 & 22.380 & 22.000 & 22.980 \\
\hline & Moment of Resistance at support & 45.480 & 44.770 & 44.000 & 45.850 \\
\hline & Shear Stress & 0.4000 & 0.4000 & 0.0000 & 0.4000 \\
\hline \multirow{5}{*}{8.} & Area of steel at mid span & 151.56 & 179.89 & 175.38 & 150.32 \\
\hline & Area of steel at support & 363.99 & 384.40 & 384.20 & 358.65 \\
\hline & Moment of Resistance at mid span & 14.550 & 21.510 & 21.000 & 14.980 \\
\hline & Moment of Resistance at support & 43.640 & 43.020 & 43.000 & 43.920 \\
\hline & Shear Stress & 0.5000 & 0.5000 & 0.0000 & 0.4500 \\
\hline \multirow{5}{*}{9.} & Area of steel at mid span & 181.88 & 188.24 & 182.40 & 180.09 \\
\hline & Area of steel at support & 378.69 & 398.22 & 394.78 & 361.00 \\
\hline & Moment of Resistance at mid span & 23.040 & 22.670 & 22.000 & 23.090 \\
\hline & Moment of Resistance at support & 46.090 & 45.350 & 45.000 & 47.020 \\
\hline & Shear Stress & 0.5000 & 0.4000 & 0.0000 & 0.4000 \\
\hline 10. & Area of steel at mid span & 213.32 & 221.60 & 218.61 & 210.65 \\
\hline
\end{tabular}


Proc. of the Eighth Intl. Conf. on Advances in Civil, Structural and Environmental Engineering - ACSEE 2019

Copyright $\odot$ Institute of Research Engineers and Doctors. All rights reserved.

ISBN: 978-1-63248-166-5 DOI : 10.15224/978-1-63248-166-5-01

\begin{tabular}{|c|c|c|c|c|c|}
\hline & Area of steel at support & 450.69 & 471.12 & 464.28 & 432.00 \\
\hline & Moment of Resistance at mid span & 30.850 & 30.390 & 30.000 & 31.030 \\
\hline & Moment of Resistance at support & 61.696 & 60.780 & 60.000 & 62.030 \\
\hline & Shear Stress & 0.5000 & 0.5000 & 0.0000 & 0.5000 \\
\hline \multirow{5}{*}{11.} & Area of steel at mid span & 218.95 & 227.20 & 225.29 & 211.00 \\
\hline & Area of steel at support & 460.95 & 480.98 & 476.66 & 455.22 \\
\hline & Moment of Resistance at mid span & 31.750 & 31.250 & 31.000 & 31.850 \\
\hline & Moment of Resistance at support & 63.500 & 62.500 & 62.000 & 63.950 \\
\hline & Shear Stress & 0.5000 & 0.4000 & 0.0000 & 0.4000 \\
\hline \multirow{5}{*}{12.} & Area of steel at mid span & 205.02 & 213.40 & 216.63 & 202.00 \\
\hline & Area of steel at support & 436.29 & 457.47 & 455.66 & 425.00 \\
\hline & Moment of Resistance at mid span & 29.500 & 29.100 & 29.000 & 29.890 \\
\hline & Moment of Resistance at support & 59.000 & 58.200 & 58.000 & 59.630 \\
\hline & Shear Stress & 0.5000 & 0.5000 & 0.0000 & 0.4000 \\
\hline \multirow{5}{*}{13.} & Area of steel at mid span & 230.00 & 227.20 & 225.29 & 225.00 \\
\hline & Area of steel at support & 435.20 & 480.98 & 476.66 & 430.00 \\
\hline & Moment of Resistance at mid span & 32.500 & 31.250 & 31.000 & 31.890 \\
\hline & Moment of Resistance at support & 65.000 & 62.500 & 62.000 & 65.060 \\
\hline & Shear Stress & 0.4000 & 0.4000 & 0.0000 & 0.4000 \\
\hline \multirow{5}{*}{14.} & Area of steel at mid span & 211.99 & 221.60 & 218.61 & 215.33 \\
\hline & Area of steel at support & 424.58 & 471.12 & 464.28 & 439.00 \\
\hline & Moment of Resistance at mid span & 31.540 & 30.390 & 30.000 & 31.600 \\
\hline & Moment of Resistance at support & 63.080 & 60.780 & 60.000 & 63.980 \\
\hline & Shear Stress & 0.4000 & 0.4000 & 0.0000 & 0.4000 \\
\hline \multirow{5}{*}{15.} & Area of steel at mid span & 193.36 & 213.40 & 212.63 & 190.00 \\
\hline & Area of steel at support & 407.82 & 457.47 & 455.66 & 400.00 \\
\hline & Moment of Resistance at mid span & 30.000 & 29.100 & 29.000 & 31.000 \\
\hline & Moment of Resistance at support & 60.000 & 58.200 & 58.000 & 61.000 \\
\hline & Shear Stress & 0.5000 & 0.5000 & 0.0000 & 0.4000 \\
\hline \multirow{5}{*}{16.} & Area of steel at mid span & 257.96 & 266.36 & 265.47 & 246.00 \\
\hline & Area of steel at support & 544.92 & 565.54 & 563.52 & 522.00 \\
\hline & Moment of Resistance at mid span & 41.760 & 41.130 & 41.000 & 41.980 \\
\hline & Moment of Resistance at support & 83.530 & 82.260 & 82.000 & 83.980 \\
\hline & Shear Stress & 0.5000 & 0.5000 & 0.0000 & 0.4000 \\
\hline \multirow{5}{*}{17.} & Area of steel at mid span & 269.54 & 277.13 & 270.70 & 275.00 \\
\hline & Area of steel at support & 564.91 & 585.48 & 578.55 & 567.00 \\
\hline & Moment of Resistance at mid span & 43.640 & 42.950 & 42.000 & 43.980 \\
\hline & Moment of Resistance at support & 87.280 & 85.910 & 85.000 & 88.010 \\
\hline & Shear Stress & 0.4000 & 0.4000 & 0.0000 & 0.4000 \\
\hline \multirow{5}{*}{18.} & Area of steel at mid span & 262.15 & 266.36 & 265.47 & 252.00 \\
\hline & Area of steel at support & 555.12 & 565.54 & 563.52 & 541.00 \\
\hline & Moment of Resistance at mid span & 41.460 & 41.130 & 41.000 & 41.980 \\
\hline & Moment of Resistance at support & 82.930 & 82.260 & 82.000 & 83.000 \\
\hline & Shear Stress & 0.5000 & 0.5000 & 0.0000 & 0.4000 \\
\hline \multirow{5}{*}{19.} & Area of steel at mid span & 255.05 & 259.23 & 252.99 & 248.00 \\
\hline & Area of steel at support & 542.24 & 552.87 & 546.31 & 525.00 \\
\hline & Moment of Resistance at mid span & 40.220 & 39.910 & 39.000 & 40.590 \\
\hline & Moment of Resistance at support & 80.440 & 79.830 & 79.000 & 80.920 \\
\hline & Shear Stress & 0.5000 & 0.5000 & 0.0000 & 0.4000 \\
\hline \multirow{5}{*}{20.} & Area of steel at mid span & 250.86 & 259.23 & 252.99 & 255.32 \\
\hline & Area of steel at support & 531.87 & 552.87 & 546.31 & 539.11 \\
\hline & Moment of Resistance at mid span & 40.500 & 39.910 & 39.000 & 41.000 \\
\hline & Moment of Resistance at support & 81.000 & 79.830 & 79.000 & 81.090 \\
\hline & Shear Stress & 0.5000 & 0.5000 & 0.0000 & 0.5000 \\
\hline \multirow{5}{*}{21.} & Area of steel at mid span & 265.20 & 273.50 & 271.19 & 262.00 \\
\hline & Area of steel at support & 558.39 & 578.71 & 573.42 & 550.00 \\
\hline & Moment of Resistance at mid span & 43.030 & 42.340 & 42.000 & 43.090 \\
\hline & Moment of Resistance at support & 86.060 & 84.690 & 84.000 & 86.090 \\
\hline & Shear Stress & 0.5000 & 0.5000 & 0.0000 & 0.5000 \\
\hline
\end{tabular}


Proc. of the Eighth Intl. Conf. on Advances in Civil, Structural and Environmental Engineering - ACSEE 2019

Copyright $(\odot$ Institute of Research Engineers and Doctors. All rights reserved.

ISBN: 978-1-63248-166-5 DOI : 10.15224/978-1-63248-166-5-01

With the help of above algorithms by collaborating it with PSO algorithm, MATLAB algorithm created to find the result by PSO approach.

\section{v. Conclusion}

From the above tabulated form results following conclusions are drawn

A. PSO method yields less area of steel as compared to other three methods i.e. manual calculations by rounding off values with IS456-2000, manual calculation without rounding off values with IS456-2000 and Excel sheet calculation though Moment of resistance for the same case by PSO method is more than other methods due to PSO algorithm.

B. Shear stress calculated by PSO method is also on safer side as compared to clauses of shear stress given by IS456-2000.

C. PSO proves to be best method for optimizing beam design with satisfying all the design clauses.

\section{References}

[1] James Kennedy and Russell Eberhart, "Particle Swarm Optimization", Proceeding of IEEE International Conference on Neural Network, Piscataway, NJ; 1995, pp. 1942-1948.

[2] Vishakha Tulsiram Holambe, Vasudev Raghunath Upadhye and Ajay Gulabrao Dahake, "Optimum Design of Singly Reinforced Beams Using Particle Swarm Optimization", Advances in civil and structural engineering, vol.1,issue12016, pp 1-10.

[3] Dian Palupi Rini, Siti Mariyam Shamsuddin and Siti Sophiyanati Yuhaniz, "Particle Swarm Optimization: Technique, System and Challenges", International Journal of Computer Applications (0975 - 8887), Volume 14 -No. 1, January 2011, pp. 19 - 27.

[4] Xiaohui Hu, Yuhui Shi and Russ Eberhart, "Recent Advances in Particle Swarm", 0-7803-85152/04/\$20.00@2004 IEEE, pp 90-97.
[5] R.E. Perez, K. Behdinan, "Particle Swarm Approach for Structural Design Optimization", Computers and Structures, Vol.85, 2007, pp. 1579-1588.

[6] Dongping Tian, Zhongzhi Shi, "Modified particle swarm optimization and it's applications", to be published.

[7] Mustafa Servet Kiran, "Particle Swarm Optimization with a New Update Mechanism", to be published.

[8] Russell Eberhart, James Kennedy, "A New Optimizer Using Particle Swarm Theory", in Proceedings of the sixth International Symposium on Micro Machine and Human Science, 1995, pp. 39-43.

[9] Y. Shi and R. C. Eberhart, "Empirical study of particle swarm optimization," in Evolutionary Computation, 1999. CEC 99. Proceedings of the 1999 Congress on, 1999, pp. 1945-1950.

[10] M. R. Tanweer, S. Suresh, N. Suresh, N. Sundararajan, "Self Regulating Particle Swarm Optimization Algorithm", to be published.

[11] Muhammad Imran, Rathiah Hashima and Noor Elaiza Abd Khalid, "An Overview of Particle Swarm Optimization Variants", Procedia Engineering 53 (2013), pp. $491-496$.

[12] X.H. Shi, Y.C. Liang, H.P. Lee, C. Lub and Q.X. Wanga, "Particle swarm optimization-based algorithms for TSP and generalized TSP”, Information Processing Letters 103 (2007), pp 169-176.

[13] A. Nimtawat and P. Nanakorn, "Simple Particle Swarm Optimization for Solving Beam-Slab layout Design Problems", Procedia Engineering 14(2011), pp 1392 1398.

[14] B. K. Chakrabarty, "A Model for Optimal Design of Reinforced Concrete Beam", J. Struct. Eng, 118(11), (1992)3238-3242.

[15] Maurice, C. (2006), Particle swarms optimization, ISTE Ltd, Great Britain and the United States.

[16] Rao, S. (1996), Engineering Optimization Theory and Practice, New Age International (p) Ltd.

[17] Deb, K. (2014), Optimization for Engineering Design Algorithms and Examples, PHI Learning private limited.

[18] Belegundu, A. (1999), Optimization Concept and Applications in Engineering, Cambridge University Press India Pvt. Ltd., Cambridge University.

[19] A. Ratnaweera, S. K. Halgamuge, and H. C. Watson, "Self-organizing hierarchical particle swarm optimizer with time-varying acceleration coefficients", IEEE Transactions on Evolutionary Computation, vol. 8, 2004, pp. 240-255.

[20] M.E.H. Pedersen and A.J. Chipper field, "Simplifying Particle Swarm Optimization", Applied Soft Computing 10 (2010), pp 618-628.

About Author:

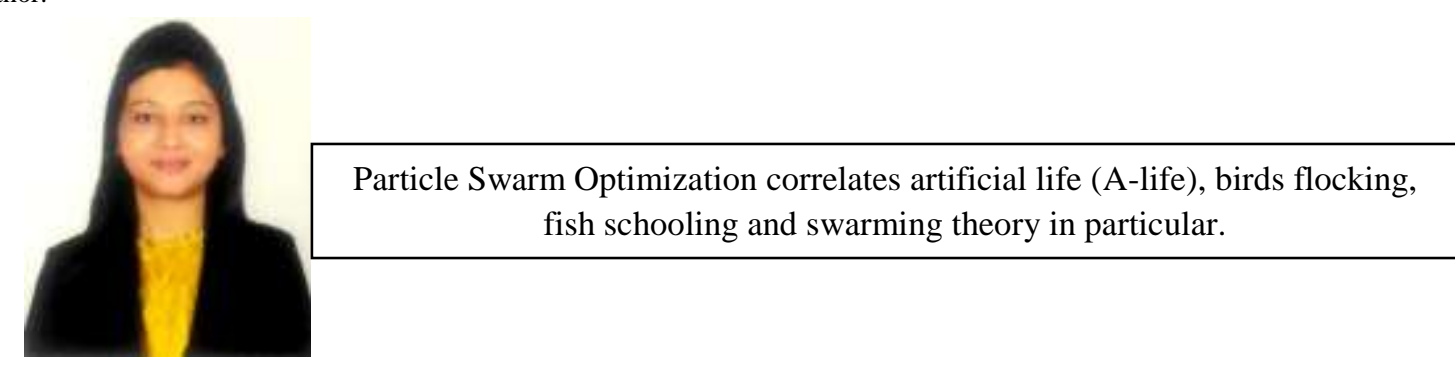

